\title{
Insônia: o benefício do exercício físico em adolescentes com excesso de peso
}

\section{Insomnia: the benefit of physical exercise in overweight teenagers}

\author{
Giselle Patrícia Pereira ${ }^{1}$, Lincoln Valério Andrade Rodrigues ${ }^{2}$, Daniella Mota Mourão ${ }^{3}$ (D) André Luiz Gomes Carneiro ${ }^{4}$, Celina \\ Aparecida Gonçalves Lima ${ }^{5}$, Carla Silvana de Oliveira e Silva ${ }^{6}$, Keila Raiany Pereira Silva@ , Claudiana Donato Bauman ${ }^{8}$
}

1. Discente do curso de Educação Física pela Universidade Estadual de Montes Claros (Unimontes), Montes Claros, MG, Brasil. 2. Discente do curso de Medicina na Universidade Estadual de Montes Claros (Unimontes), Montes Claros, MG, Brasil. 3. Docente do curso de Medicina, Doutora pelo Instituto Universitário Italiano de Rosário (UNIR), Argentina. 4. Dcente do curso de Educação Física na Universidade Estadual de Montes Claros (UNIMONTES), Montes Claros, MG, Brasil, Douror pela Universidade Tras-dos-Montes e Alto Douro,Portugual.5. Docente do Departamento Ciências Exatas, Mestre pela Universidade Estadual de Montes Claros (Unimontes),MG, Brasil. 6. Docente do curso de Enfermagem na Universidade Estadual de Montes Claros (Unimontes), MG, Brasil. 7 Discente do curso de Medicina na Universidade Estadual de Montes Claros (Unimontes), Montes Claros, MG, Brasil. 8. Docente do curso de Educação Física na Universidade Estadual de Montes Claros (Unimontes), Montes Claros, MG, Mestre pela Universidade Estadual de Montes Claros (Unimontes), Montes Claros, MG, Brasil.

\section{Resumo}

Introdução: O sono é uma necessidade vital e fisiológica que tem o poder de restauração do organismo e conservação da energia. Entre os adolescentes, notou-se que houve uma diminuição nas horas de sono tornando-os muito vulneráveis à apresentação do quadro de insônia. Objetivo: Verificar níveis de insônia entre adolescentes com excesso de peso antes e depois de um programa de exercício físico. Métodos: Trata-se de um estudo epidemiológico quase experimental e analítico. Na primeira fase do estudo, foram realizados exames laboratoriais, antropométricos, hemodinâmicos e análises nutricionais e psicológicas em 635 adolescentes com idade entre 10 a 16 anos. Destes, 63 adolescentes foram diagnosticados com exceso de peso sendo convidados a participarem de um programa de exercício fisico. Resultados: 0 grupo-controle evidenciou maior percentual (acima da média) de insônia, tanto no pré (72,7\%), quanto no pós teste (90\%), e na comparação das médias dos dois grupos, houve uma diferença significativa $(p=0,006)$. Conclusão: Ressalta-se a significativa diminuição nos níveis de insônia entre os adolescentes que participaram do programa de exercício, propiciando maior bem-estar e disposição para o dia a dia.

Palavras-chave: Insônia. Adolescentes. Excesso de peso.

\begin{abstract}
Introduction: Sleep is a vital and physiological necessity that has the power to restore one's organism and to conserve one's energy. Among teenagers, it was noticed that there was a decrease in sleep hours, making them become vulnerable to the insomnia state. Objective: To verify the levels of insomnia among teenagers before and after the participation in a physical exercise program. Methods: This research is an epidemiological study, almost experimental and analytical. In the first stage, there were promoted laboratory exams, anthropometric, hemodynamic, and nutritional and psychological analysis for 635 teenagers aged between 10 to 16 years. Among them, 63 teenagers were diagnosed with overweight and obesity, so they were invited to participate in a physical exercise program. Results: The control group showed a bigger percentage of insomnia (above average), in both the pre-test (72.7\%) and in the post-test $(90 \%)$, and in the average comparison of the two groups there was a significant difference $(p=0.006)$. Conclusion: A significant decrease of the levels of insomnia among teenagers that participated in the exercise program was noted, providing greater well-being and mood for their daily activities.
\end{abstract}

Key words: Insomnia. Teenagers. Overweight.

\section{INTRODUÇÃO}

O sono é uma necessidade vital e fisiológica que detém o poder de restauração do organismo e conservação da energia para a obtenção de uma boa saúde, disposição física, mental e cognitiva. A necessidade de um adolescente de 11 a 17 anos varia entre oito a nove horas de sono e é recomendado nos dias com aula uma noite de sono de pelo menos 8,33 horas ${ }^{1,2,3}$. A qualidade do sono pode diminuir a qualidade de vida e acarretar redução do rendimento escolar de crianças e adolescentes, resultando em importantes consequências para a saúde desta população ${ }^{4}$.

A insônia é uma síndrome definida como dificuldade em iniciar e/ou manter o sono, presença de sono não reparador, com consequente comprometimento do desempenho nas atividades diurnas, sendo um importante distúrbio em crianças e adolescentes e essencial para manter uma boa qualidade de alerta e bem-estar físico e mental durante o dia ${ }^{5}$. É classificada como primária ou secundária, sendo a primária caracterizada por inúmeros sintomas e possui uma capacidade relativamente simples de resposta geral ao tratamento, enquanto a secundária é o resultado de outras doenças, do uso de medicamentos ou de outros distúrbios primários do sono. Pode ser dividida em inicial, quando as queixas estão relacionadas às dificuldades de adormecer. A insônia intermediária diz respeito ao sono interrompido e quando se tem dificuldade em retomar o sono e, por último, a insônia terminal é caracterizada pelo despertar

Correspondência: Giselle Patrícia Pereira. Universidade Estadual de Montes Claros (Unimontes), Montes Claros, MG, Brasil. Avenida Dr. Ruy Braga, S/N Vila Mauriceia, Montes Claros - MG, 39401-089. e-mail: gisellepatricia04@gmail.com

Conflito de interesse: Não há conflito de interesse por parte de qualquer um dos autores.

Recebido em: 8 Fev 2018; Revisado em: 5 Abr 2018; 21 Maio 2018; Aceito em: 24 Jul 2018 
precoce, sem que se consiga retomar o sono ${ }^{6}$.

Entre os adolescentes tem-se constatado uma diminuição nas horas de sono, apesar da necessidade para manterem o bem estar, a saúde e o desenvolvimento através do hormônio do crescimento $(\mathrm{GH})$. Alguns adolescentes não têm o hábito de sono regular, ficando acordados até tarde durante a semana e buscando a compensação nos finais de semana, portanto se tornam muito vulneráveis à apresentação do quadro de insônia ${ }^{1,6}$.

Devido às alterações biológicas que ocorrem na adolescência, o quesito sono-vigília ao se manter irregular pode acarretar uma sonolência diurna excessiva, assim como insônia. Entre os principais fatores associados aos distúrbios do sono podese citar a condição socioeconômica, psicológica, física e ambiental, além da era tecnológica que vem causando grandes transformações na vida contemporânea, pois os adolescentes tendem a ficar conectados às diversas tecnologias do mundo globalizado, quase toda a noite $e^{5,6}$.

As consequências da privação do sono são diversas e estão relacionadas à redução no tempo de reação e eficiência no desempenho cognitivo e motor, aumento da irritabilidade, alterações metabólicas, endócrinas, imunológicas, quadros hipertensivos e dores articulares. A carência do sono diminui o metabolismo na região frontal e no cerebelo, responsáveis pelo planejamento e pela execução das tarefas, e pelo centro de coordenação motora que acarreta alterações de humor, atenção, memória e equilíbrio ${ }^{6,7}$.

A saúde física e mental está relacionada a uma qualidade do sono com excelência; quando isso não ocorre, aumenta a possibilidade do desenvolvimento de algumas doenças, como: obesidade, diabetes, problemas no sistema cardiovascular e respiratório ${ }^{1}$. Vários estudos demonstram associação entre o ciclo sono-vigília e os hormônios reguladores do apetite, como a leptina e a grelina, sendo que o primeiro estimula a saciedade e o segundo estimula o apetite, gerando um desequilíbrio metabólico que resulta em excesso de peso ${ }^{5,8,9}$. Na privação do sono, há elevação dos níveis de grelina e diminuição da leptina, com consequente aumento da ingestão alimentar e da fome, preferencialmente por alimentos hipercalóricos.

Atualmente, o excesso de peso (sobrepeso e obesidade) atinge cifras alarmantes em todo o mundo, resultando em alterações endócrinas e metabólicas na infância e na adolescência, com complicações cardiovasculares na vida adulta, como infarto miocárdio e acidente vascular cerebral. O aumento de sua prevalência, consequente da mudança do estilo de vida na população decorrido do aumento do sedentarismo e da alta ingestão calórica, gera preocupação e tornou-se um desafio para a saúde pública ${ }^{4}$.

Nesta perspectiva, a prática do exercício físico desempenha um papel fundamental sobre a saúde e a aptidão física do indivíduo. No que diz respeito à prática do exercício e à qualidade do sono, existem duas hipóteses que sustentam positivamente seus benefícios; a primeira, consiste no aumento da temperatura corporal que facilita o disparo do mecanismo do sono e a segunda seria o aumento do gasto energético que resulta em uma maior necessidade de sono para o alcance de um mecanismo homeostático positivo ${ }^{10}$.

O exercício físico regular propicia uma melhor capacidade cardiorrespiratória, força muscular, flexibilidade, desenvolvimento e fortalecimento da massa óssea, resistência de tendões e ligamentos, aumento do metabolismo em repouso, melhoria da função imunitária, produção de serotonina, controle do peso corporal, melhora cognitiva, consequentemente melhora na qualidade do sono ${ }^{11}$. Como alternativa de auxiliar no tratamento da insônia, o exercício físico gera um aumento nas horas de sono a fim de o corpo recuperar a homeostasia modificada por tal, o que pode ser notado tanto em exercícios agudos quanto crônicos. O ciclo sono-vigília também sofre modificações no tocante ao relógio biológico quando há uma melhora na qualidade do sono e a sincronização dos horários de sono na melhor hora do dia ${ }^{12}$.

São limitados na literatura os artigos que relacionam os benefícios do exercício físico na insônia em adolescentes com excesso de peso. Nessa perspectiva, o presente estudo teve como objetivo verificar os níveis de insônia entre adolescentes com excesso de peso antes e depois de um programa sistematizado de exercícios físicos.

\section{MÉTOdOS}

\section{Tipo do estudo}

Trata-se de um estudo epidemiológico de caráter quase experimental e analítico, desenvolvido com base no projeto "Influência de um programa de atividade física em adolescentes com risco cardiovascular", com o objetivo de avaliar o estado nutricional, nível socioeconômico, atividade física, depressão, qualidade de vida, saúde bucal e associação com síndrome metabólica e obesidade em escolares de Montes Claros, Minas Gerais, Brasil.

\section{População}

A população foi selecionada por conglomerados probabilísticos, quando se dividiu a cidade de Montes Claros (MG) em quatro regiões: norte, sul, leste e oeste. Após a listagem das 63 escolas públicas, quantificou-se o número de alunos matriculados totalizando-se 77.833, que foram estratificados por idade. Para a representação da população total, empregou-se o erro padrão de 0,15 e com uma probabilidade de ocorrência de $50 \%$. A primeira fase do estudo foi composta por 635 adolescentes com idades entre 10 a 17 anos que realizaram exames laboratoriais, antropométricos, hemodinâmicos e análises nutricionais e psicológicas. 


\section{Amostra}

Identificaram-se 63 escolares com excesso de peso, sendo que na segunda fase foram convidados a participar de um programa sistematizado de exercício físico. Para este estudo, 22 adolescentes aceitaram participar e 41 recusaram. Entre os 22 escolares, nove adolescentes compuseram o grupo de intervenção e 13 o grupo controle.

\section{Critérios de inclusão e exclusão}

Foram incluídos os adolescentes devidamente matriculados na rede pública estadual de ensino, com excesso de peso segundo critérios da Organização Mundial da Saúde ${ }^{13}$, que assinaram o Termo de Assentimento Livre e Esclarecido (TALE), assim como o Termo Consentimento Livre e Esclarecido (TCLE) assinados pelos responsáveis. Neste estudo, foram excluídas as adolescentes grávidas, aqueles em uso de medicamentos que influenciavam no perfil metabólico e/ou hemodinâmico e portadores de doenças renais, inflamatórias, infecciosas, hepáticas e hematológicas significativas.

\section{Calibração da equipe}

A equipe de profissionais e acadêmicos de educação física foi treinada e calibrada para realizar a intervenção, sob a supervisão de um coordenador responsável. A coleta dos dados foi feita antes e após a intervenção. Para a aplicabilidade do índice de gravidade da insônia, os resultados foram submetidos ao coeficiente Kappa e o grau de concordância foi de 0,85 (inter-examinador) e 0,81 (intra-examinador). Com relação às avaliações antropométricas (peso, altura, circunferência da cintura), os resultados foram de 0,94 e 0,96 (inter e intraexaminador), respectivamente.

\section{Questionário}

Para a avaliação da insônia, foi usado o Índice de Gravidade da Insônia (IGI) composto por sete itens com opções de respostas de zero a quatro breves e simples que objetivam identificar a percepção da gravidade da insônia (leve, moderada e grave). O índice varia zero a 28 pontos, sendo que escore de zero a sete indica insônia clinicamente significante; oito a 14 insônia subliminar; 15 a 21 insônia moderada e de 22 a 28 insônia grave $^{15}$.

\section{Procedimentos}

Os escolares realizaram avaliação física no início e no final da intervenção. Para determinar a intensidade do treinamento de força foi realizado o teste de carga com o protocolo Brzycki e em seguida foi planejada a ficha individual de cada adolescente determinada pelos resultados ${ }^{16}$. 0 programa de exercício físico foi realizado três vezes por semana por 60 minutos durante 12 semanas. $O$ treinamento consistia em 10 minutos de esteira no início e no final de cada sessão, com 40 minutos de treinamento de força e posterior relaxamento. O questionário de insônia foi aplicado antes e após a participação dos adolescentes no programa de exercício físico pré-estabelecido.

\section{Considerações éticas}

O estudo foi aprovado pelo Comitê de Ética em Pesquisa da Universidade Estadual de Montes Claros (Unimontes), sob o parecer consubstanciado $\mathrm{n}$ - 1.503.680. Os responsáveis e os adolescentes foram informados a respeito dos objetivos do estudo, sendo também assegurado o anonimato dos participantes.

Análise de dados

As variáveis analisadas foram descritas por frequências absolutas e relativas. Para comparar a diferença entre as médias, foi realizado o teste $t$ de Student para amostras independentes, sendo considerado estatisticamente significativo $p \leq 0,05$. Todas as análises estatísticas foram realizadas utilizando-se o programa SPSS (Statistical Package for Social Science), versão 20.0 para Windows.

\section{RESULTADOS}

Na Tabela 1 apresentou-se a caracterização da amostra relacionando variáveis sociodemográficas, grupos de intervenção e controle, relato de doenças, uso de medicação, consumo de bebidas alcoólicas e prática de atividade física de adolescentes de Montes Claros - MG, 2017. Os 22 adolescentes participantes do presente estudo tiveram idade média de 13,4 $\pm 1,7$ anos. O IMC médio da população foi de $28,5 \pm 3,1$. O nível de escolaridade que apresentou maior prevalência foi o ensino fundamental que corresponde do $6^{\circ}$ ao $9^{\circ}$ ano $(66,7 \% ; n=$ 15). A maioria dos adolescentes se declarou pardos $(52,4 \%)$ e católicos (63,6\%).

Tabela 1. Caracterização da amostra dos adolescentes das escolas públicas de Montes Claros - MG, 2017.

\begin{tabular}{|c|c|c|}
\hline Variáveis & $\mathbf{n}$ & $\%$ \\
\hline \multicolumn{3}{|l|}{ Gênero } \\
\hline Masculino & 11 & 50,0 \\
\hline Feminino & 11 & 50,0 \\
\hline \multicolumn{3}{|l|}{ Faixa etária } \\
\hline 10-11 anos & 2 & 9,0 \\
\hline $12-13$ anos & 10 & 45,4 \\
\hline 14-15 anos & 6 & 27,2 \\
\hline $16-17$ anos & 4 & 18,2 \\
\hline \multicolumn{3}{|l|}{ Grupos } \\
\hline Intervenção & 9 & 40,9 \\
\hline Controle & 13 & 59,1 \\
\hline \multicolumn{3}{|c|}{ Presença de doenças } \\
\hline Não & 19 & 86,4 \\
\hline Sim & 3 & 13,6 \\
\hline \multicolumn{3}{|c|}{ Uso de medicações } \\
\hline Não & 18 & 81,8 \\
\hline Sim & 4 & 18,2 \\
\hline
\end{tabular}




\begin{tabular}{lrr}
\hline Variáveis & $\mathbf{n}$ & $\mathbf{\%}$ \\
\hline Uso de bebidas alcoólicas & & \\
\hline Não & 19 & 86,4 \\
Sim & 3 & 13,6 \\
\hline Renda familiar mensal & & \\
Até 3 salários mínimos & 17 & 81,0 \\
3 a 10 salários mínimos & 04 & 19,0 \\
Prática de exercícios físicos & & \\
\hline Não & 13 & 59,1 \\
Sim & 9 & 40,9 \\
\hline Fonte: Dados da pesquisa.
\end{tabular}

Fonte: Dados da pesquisa.

No que diz respeito à comparação entre os grupos antes e após a prática do programa de exercício físico, os resultados demonstraram que o grupo controle exibiu um percentual maior (acima da média) de insônia, tanto no pré, quanto no pós-teste (Tabela 2).

Tabela 2. Comparação entre os grupos antes e após a prática de exercícios físicos dos adolescentes das escolas públicas de Montes Claros - MG, 2017.

\begin{tabular}{llll}
\hline \multirow{2}{*}{ Insônia } & \multicolumn{3}{c}{ Grupos } \\
\cline { 2 - 4 } & $\begin{array}{c}\text { Intervenção } \\
\mathrm{n}(\%)\end{array}$ & $\begin{array}{c}\text { Controle } \\
\mathrm{n}(\%)\end{array}$ & $\begin{array}{c}\text { Total } \\
\mathrm{n}(\%)\end{array}$ \\
\hline Insônia pré-teste & & & \\
\hline Insônia não significativa & $6(54,5)$ & $5(45,5)$ & 11 \\
Insônia & $3(27,3)$ & $8(72,7)$ & 11 \\
Insônia pós-teste & & & \\
Insônia não significativa & $8(66,7)$ & $4(33,3)$ & 12 \\
Insônia/Insônia moderada & $1(10,0)$ & $9(90,0)$ & 10 \\
\hline Fonte:
\end{tabular}

Fonte: Dados da pesquisa.

Com relação às médias de insônia do grupo intervenção e controle, nota-se que houve uma diferença significativa entre os adolescentes que participaram do programa de exercício físico quando comparados com o grupo controle (Tabela 3).

Tabela 3. Média de insônia dos adolescentes das escolas públicas de Montes Claros - MG, antes e após programa de exercícios físicos, 2017.

\begin{tabular}{llllll}
\hline Variável & Grupos & N & Média & $\begin{array}{l}\text { Desvio } \\
\text { padrão }\end{array}$ & Valor p \\
\hline Insônia pré-teste & Intervenção & 9 & 5,0 & 4,1 & 0,142 \\
& Controle & 13 & 7,6 & 3,9 & \\
\multirow{2}{*}{ Insônia pós-teste } & Intervenção & 9 & 3,7 & 3,4 & $0,006^{*}$ \\
& Controle & 13 & 8,8 & 4,1 & \\
\hline
\end{tabular}

Fonte: Dados da pesquisa

\section{DISCUSSÃO}

A insônia na juventude está associada a fatores demográficos, biopsicossociais, ambientais e a hábitos de vida, como o consumo de produtos ricos em cafeína e de bebidas alcoólicas, sedentarismo, tabagismo e à alimentação ${ }^{17,18}$.

Com relação aos fatores demográficos, o estudo de Johnson et al. (2006) demonstrou que a prevalência de insônia aumentou com a idade e está relacionado com o baixo nível socioeconômico dos adolescentes ${ }^{19}$. Corroborando estes resultados verificou-se que $81 \%$ da amostra possuía renda de até três salários mínimos. Siomos (2010) também relatou associação entre os distúrbios de sono e o status socioeconômico, visto que as famílias menos favorecidas economicamente apresentam habitações menos organizadas, com menor consciência da importância da higiene do sono e com maiores índices domiciliares de ruído, o que afetaria negativamente a qualidade do sono ${ }^{20}$.

Entre os hábitos de vida, o consumo de bebidas alcoólicas pelos adolescentes é importante fator relacionado aos distúrbios de sono. Estudo realizado por Coutinho (2016) evidencia que as dificuldades para iniciar e manter o sono, assim como o sono insuficiente são frequentes entre os jovens, podendo acarretar transtornos de consumo excessivo de bebidas alcoólicas ${ }^{21}$. No presente estudo, a maioria dos adolescentes relatou não consumir bebidas alcoólicas $(86,4 \%)$; apesar disso, foi alto o percentual de insônia na população estudada, demonstrando a necessidade de novos estudos a fim de elucidar outras causas e/ou associações da insônia nesta população.

O presente estudo verificou que o grupo submetido ao programa de exercício físico apresentou um menor percentual de insônia antes e após a realização do programa pré-estabelecido, em comparação ao grupo controle. Corroborando com isso o estudo de Chen et al.(2008) evidenciou conexão bidirecional entre o sono e o exercício físico: quanto maior a prática de exercício físico, maior a duração e qualidade do sono, sendo também que indivíduos com perturbação da duração e qualidade do sono estão associados a um maior grau de sedentarismo devido à fadiga e outros fatores ainda pouco elucidados ${ }^{22}$. No presente estudo, o grupo controle exibiu percentual maior de insônia tanto no pré $(72,7 \%)$ como pós-teste $(90,0 \%)$, evidenciado piora da insônia nos adolescentes sedentários.

Desde 1966 Baekeland e Lasky já comprovavam associação positiva entre o exercício físico realizado durante o dia e a quantidade de ondas lentas nos estágios três e quatro do sono em dez jovens do sexo masculino por meio da polissonografia após sessão rotineira de exercícios físicos no período vespertino ${ }^{23}$. Porém a National Sleep Foundation já evidenciava a existência de poucas investigações sobre o efeito do exercício físico na insônia, e mais limitadas ainda, são as pesquisas desses benefícios em adolescentes com excesso de peso ${ }^{24}$. A 
fim de auxiliar maiores esclarecimentos sobre o assunto este estudo mostrou que o índice de insônia no grupo intervenção foi menor que aquele apresentado no controle, com diferença significativa $(p=0,006)$.

O exercício físico desempenha papel essencial sobre a condição física, psicológica e mental em crianças e adolescentes, beneficiando a qualidade do sono. O estudo de Bois et al. (2005), salientou que o exercício físico pode aumentar a autoestima, a aceitação social e a sensação de bem-estar entre as crianças, influindo positivamente no sono ${ }^{25}$. Também Biazussi (2017) ressaltou o impacto favorável do exercício físico no crescimento e desenvolvimento do adolescente, possibilitando a promoção da socialização, melhora da motricidade, da flexibilidade e do autoconhecimento corporal, bem como a elevação da autoestima e prevenção de doenças associadas à obesidade, fatores importantes para adequada qualidade do sono ${ }^{17}$.

A prática de exercício físico é reconhecida como uma intervenção não farmacológica que melhora o padrão e a qualidade do sono ${ }^{26}$. Ainda assim, a prescrição de exercícios físicos com esse objetivo é reduzida, provavelmente devido ao desconhecimento por parte dos educadores físicos e médicos sobre benefícios do exercício físico nos distúrbios do sono.

Ressalta-se, ainda, que a maioria dos estudos epidemiológicos relacionados ao sono na adolescência foi realizada nos Estados Unidos e na Europa, sendo que são escassos os estudos sobre os distúrbios do sono na população brasileira. Também na literatura, são poucos os estudos que demonstram o benefício da prática de exercício físico em adolescentes com excesso de peso portadores de insônia, como o presente estudo ${ }^{27}$.

\section{CONCLUSÃO}

Verificou-se que houve diminuição dos níveis de insônia entre os adolescentes que participaram do programa de exercícios físicos. O exercício físico desempenhou papel fundamental, como uma medida não farmacológica, propiciando resultados positivos na qualidade de vida dos adolescentes. O presente estudo possibilita o despertar de novos rumos de investigação, que permite contribuir para um melhor esclarecimento de aspectos que carecem de maior investigação, particularmente em relação aos benefícios da prática de exercício físico sobre o sono, nesta população.

\section{REFERÊNCIAS}

1. Seixas MP. Avaliação da qualidade do sono na adolescência: implicações para a saúde física e mental [dissertação]. Porto: Universidade Fernando Pessoa; 2009.

2. Pereira CM. Alterações do sono no sistema cardiovascular. [dissertação]. Covilhã: Universidade da Beira Interior; 2009.

3. Pereira EF, Barbosa DG, Andrade RD, Claumann GS, Pelegrini A, Louzada FM. Sono e adolescência: quantas horas os adolescentes precisam dormir? J bras psiquiatr [internet].2015 Mar [acesso 2017 maio 10]; 64(1):40-44. Disponível em: http://www.scielo.br/pdf/jbpsiq/v64n1/0047-2085-jbpsiq-64-1-0040.pdf. doi: 10.1590/0047-208500000005.

4. Souza LTN, Tomaz RR. Qualidade de sono, qualidade de vida e rendimento escolar de crianças no litoral sul da Paraíba. J Health Biol Sci. [internet].2018 Jan-Mar [acesso 2018 Abr 12]; 6(1): 42-47.

5. Turco GF, Reimão R, Rossini S, Antonio MARGM, Barros AA Filho. Distúrbios do sono e qualidade de vida em crianças e adolescentes obesos - Revisão Bibliográfica. Neurobiologia. 2011 Abr-Jun;72(2):171-180.

6. Amaral MOP. Epidemiologia da insónia em adolescentes do diagnóstico de situação à intervenção [tese]. Lisboa: Universidade Nova de Lisboa; 2013.

7. Ciampo LA. O sono na adolescência. Adolesc Saúde [internet]. 2012 Abr-Jun [acesso 2017 Maio 10]; 9(2):60-66. Disponível em: http://www. adolescenciaesaude.com/detalhe artigo.asp?id=317.

8. Antunes HKM, Andersen ML, Tufik S, Mello MT. Privação de sono e exercício físico. Rev. Bra Med Esporte [Internet]. 2008 Jan-Fev [acesso 2017 Maio 10];14(1):55-56. Disponível em: http://www.scielo.br/scielo.php?script=sci_ arttext\&pid=S1517-86922008000100010.

9. Crispim CA, Zalcman I, Dáttilo M, Padilha HG,Tufik S, Mell MT. Relação entre sono e obesidade: uma revisão da literatura. Arq Bras Endocrinol Metab. 2007 Out; 51(7):1041-1049. doi: http://dx.doi.org/10.1590/S000427302007000700004 .
10. Gibbert GA. Brito MN. Relações fisiológicas entre o sono e a liberação de hormônios que regulam o apetite. Rev Saúde Pesq. 2011; 4(2): 271-277.

11. Silva PVC, Costa AL Jr. Efeitos da atividade física para a saúde de crianças e adolescentes. Psicol Argum [internet]. 2011 Jan-Mar [acesso 2017 maio 11]; 29(64): 41-50. Disponível em: http://repositorio.unb.br/handle/10482/20614. 12. Boscolo RA, Sacco IC, Antunes HK, Mello MT, Tufik S. Avaliação do padrão de sono, atividade física e funções cognitivas em adolescentes escolares. Rev Port Cien Desp. 2007 Jan; 7(1): 18-25.

13. Onis M, Onyango AW, Borghi E, Siyam A, Nishida C, Siekmann J. Development of a WHO growth reference for school-aged children and adolescents. Bull World Health Organ. 2007 Sep; 85(9): 660-667. PubMed PMID: 18026621.

14. Sim J, Wright CC. The kappa statistic in reliability studies: use, interpretation, and sample size requirements. Phys Ther. 2005 Mar; 85(3): 257-268.

15. Gorestein C, Tavares S, Alóe F. Questionários de auto-avaliação de sono. In: Gorestein C, Andrade LHS, Zuard AW. Escalas de avaliação clínica em psiquiatria e psicofarmacologia. São Paulo: Lemos; 2000.

16. Brzycki M. Strength testing: predicting a one-rep max from repetitions to fatigue. JOPERD. 1993; 64(1): 88-90. doi: https://doi.org/10.1080/07303084.1 993.10606684

17. Biazussi R. Os benefícios da atividade física aos adolescentes. [internet]. 2008 [acesso 2017 Dez. 27].13-18 Disponível em: http://www.saudecomaventura. com.br/dicasaventureiro/Beneficio1.PDF.

18. Reimão R. Sono normal e seus distúrbios na criança. In: Diament A, Cypel S. Neurologia Infantil. 4. ed. São Paulo. Atheneu; 2005. p.1335-1342.

19. Johnson EO, Roth T, Schultz L, Breslau N. Epidemiology of DSM-IV insomnia in adolescence: lifetime prevalence, chronicity, and an emergent gender difference. Pediatrics. 2006 Fev; 117(2):e247-256. doi:10.1542/peds.20042629.

20. Siomos KE, Avagianou P-A, Floros GD, Skenteris N, Mouzas OD, Theodorou 
$\mathrm{K}$, et al. Psychosocial correlates of insomnia in an adolescent population. Child Psychiatry Hum Dev. 2010 Jun; 41(3):262-73. doi: 10.1007/s10578-009-0166-5.

21. Coutinho ESF, França-Santos D, Magliano ES, Bloch KV, Barufaldi LA, Cunha CF, et al. ERICA: Padrões de consumo de bebidas alcoólicas em adolescentes brasileiros. Rev Saúde Pública [internet]. 2016 Out [acesso 2018 Abr 17]; 50(supl 1): 8s. Disponível em: http://www.scielo.br/pdf/rsp/v50s1/ pt_0034-8910-rsp-S01518-87872016050006684.pdf. doi:10.1590/S01518 8787.2016050006684 .

22. Chen X, Beydoun MA, Wang Y. Is sleep duration associated with childhood obesity? A systematicreviewand meta-analysis. Obesity (Silver Spring) 2008 Feb;16(2): 265-274. doi:10.1038/oby.2007.63.

23. Baekeland F, Lasky R. Exercise and sleep patterns in college athletes. Percep
Mot Skills.1966 Dec; 23(3):1203-1207. PubMed PMID: 5972920.

24. National Sleep Foundation. How does exercise help those with chronic insomnia? [internet]. 2013 [acesso 2017 Dec 27]. Disponível em: http://www. sleepfoundation.org/article/howdoes-exercise-help-those-chronic-insomnia.

25. Bois JE, Sarrazin PG, Brustad RJ, Trouilloud DO, Cury F. Elementary schoolchildren's perceived competence and physical activity involvement: The influence of parents' role modeling behaviors and perceptions of their child's competence. Psychol Sport Exerc. 2005; 6(4):381-397.

26. Lavie P. The enchanted world of sleep. New Haven: Yale University Press; 1996.

27. Jacinto CC. Sono e Obesidade em idade pediátrica [dissertação]. Lisboa: Faculdade de Medicina da Universidade de Lisboa; 2016. 